\title{
A simple method for calculating the statistical power for detecting a QTL located in a marker interval
}

\author{
$\mathrm{Z} \mathrm{Hu}$ and $\mathrm{S} \mathrm{Xu}$ \\ Department of Botany and Plant Sciences, University of California, Riverside, CA, USA
}

\begin{abstract}
We developed a simple method for calculating the statistical power for detecting a QTL located in an interval flanked by two markers. The statistical method for QTL detection is assumed to be the Haley and Knott's simple regression method of interval mapping. This method allows us to answer one of the fundamental questions in designing a QTL mapping experiment: What is the minimum marker density required to detect a QTL explaining a certain
\end{abstract}

Keywords: QTL mapping; quantitative trait; statistical power

\section{Introduction}

Simple methods for calculating the statistical power for detecting a QTL that overlaps with a marker have been developed (Soller et al., 1976; Muranty, 1996). When the QTL of interest is located further away from a marker, which is used to detect the QTL, the power will be decreased (Soller and Genizi, 1978; Lynch and Walsh, 1998). Simple methods for calculating the statistical power for a QTL located in an interval flanked by two markers have not been available. Statistical methods for power calculation based on the likelihood ratio test statistic using markers of the entire genome (interval mapping) have been developed by Dupuis and Siegmund (1999). However, the methods depend on some assumptions such as infinitely high marker density or equal distance of marker distribution, and thus they can be very complicated. Simple regression method for QTL mapping (Haley and Knott, 1992) is a good approximation of the maximum likelihood method. Therefore, statistical power may be calculated based on the simple regression method. Because of the simplicity of the regression method, calculation of statistical power under this method is also simple. If the QTL of interest does not overlap with a marker, the power of detecting this QTL will be decreased. The worst-case scenario is that the QTL sits in the middle of the largest marker interval. If we can compute the statistical power for detecting such a QTL, the inverse power function will allow us to design a QTL mapping experiment regarding the sample size and the minimum marker density

Correspondence: Professor Shizhong Xu, Department of Botany and Plant Sciences, University of California, Riverside, CA 92521, USA.

E-mail: shizhong.xu@ucr.edu

Received 29 November 2007; revised 18 February 2008; accepted 17 March 2008; published online 30 April 2008 heritable proportion of the phenotypic variance (denoted by $h^{2}$ ) with a power $\gamma$ under a Type I error $\alpha$ in an $F_{2}$ or other mating designs with a sample size $n$ ? Computing the statistical power only requires the ability to evaluate a noncentral F-distribution function and the inverse function of this distribution.

Heredity (2008) 101, 48-52; doi:10.1038/hdy.2008.25; published online 30 April 2008

required. This article will introduce such a simple method.

\section{Methods}

We now use an $\mathrm{F}_{2}$ mating design as an example to develop the method. Extension to other mating types will be described later in the Discussion section. Although $t$-test is commonly used for the simple regression analysis, F-test is used here to discuss the power calculation because we can avoid the confusion between one-tailed and two-tailed tests that occur in the $t$-test. With the F-test, only a single tail of the distribution is concerned. In addition, when only additive effect is considered, the F-test statistic is simply the square of the $t$-test statistic. Let $A_{1} A_{1}, A_{1} A_{2}$ and $A_{2} A_{2}$ be the three genotypes of a QTL in an $\mathrm{F}_{2}$ family. Let

$$
x_{j}=\left\{\begin{array}{rll}
+1 & \text { for } & A_{1} A_{1} \\
0 & \text { for } & A_{1} A_{2} \\
-1 & \text { for } & A_{2} A_{2}
\end{array}\right.
$$

be the genotype indicator variable for individual $j$ for $j=1, \ldots n$, where $n$ is the sample size. Let $a$ be the additive genetic effect (the difference between the genotypic values of $A_{1} A_{1}$ and $A_{1} A_{2}$ ). Assume that the QTL of interest overlaps with a fully informative marker, the test statistic under the regression analysis is

$$
\lambda=\sum_{j=1}^{n}\left(x_{j}-\bar{x}\right) \frac{\hat{a}^{2}}{\hat{\sigma}^{2}}
$$

where â is the least square estimate of the genetic effect and $\hat{\sigma}^{2}$ is the estimated residual error variance. Under the null hypothesis $(a=0)$, the above test statistic will follow a central F-distribution with degrees of freedom 1 and $n$ (assuming that $n$ is sufficiently large). Under the 
alternative hypothesis $(a \neq 0)$, the test statistic will follow a non-central F-distribution with degrees of freedom 1 and $n$ and a non-centrality parameter

$$
\delta=n \sigma_{x}^{2} \frac{a^{2}}{\sigma^{2}}
$$

where $\sigma_{x}^{2}=1 / 2$ is the variance of variable $x$ across all individuals within the $\mathrm{F}_{2}$ family (assuming that there is no segregation distortion). Let $F(\lambda \mid 1, n, 0)$ and $F(\lambda \mid 1, n$, $\delta$ ) be the central and non-central F-distribution functions for variable $\lambda$, respectively. Define $\lambda_{1-\alpha}$ as the $1-\alpha$ percentile of the central F-distribution, that is, $\alpha=\operatorname{Pr}\left(\lambda>\lambda_{1-\alpha} \mid \delta=0\right)=1-F\left(\lambda_{1-\alpha} \mid 1, n, 0\right)$ or

$$
\lambda_{1-\alpha}=F^{-1}(1-\alpha \mid 1, n, 0)
$$

where $\alpha$ is the Type I error. The Type II error is defined as $\beta=\operatorname{Pr}\left(\lambda \leqslant \lambda_{1-\alpha} \mid \delta>0\right)$, that is,

$$
\beta=F\left(\lambda_{1-\alpha} \mid 1, n, \delta\right)
$$

The statistical power is

$$
\gamma=1-\beta
$$

To calculate the statistical power for a given Type I error $\alpha$, we first use equation (4) to find the critical value $\lambda_{1-\alpha}$ and then use equation (5) to calculate the Type II error $\beta$, and finally use equation (6) to get the statistical power $\gamma$.

The three steps used to calculate the statistical power is general for all experiments using the $F$-test statistic. The non-centrality parameter $\delta$, however, depends on the experimental parameters of the specific experiment. In addition, if the QTL of interest does not overlap with a marker, the non-centrality parameter should be revised to take into account the linkage information between the QTL and markers.

Let $m_{1}$ and $m_{2}$ be the genotype indicator variables (similarly defined as variable $x$ ) for two flanking markers. Let $r_{1}$ and $r_{2}$ be the recombination fractions between $m_{1}$ and $x$ and between $x$ and $m_{2}$, respectively. The recombination fraction between $m_{1}$ and $m_{2}$ is $r_{12}=r_{1}+r_{2}-2 r_{1} r_{2}$. Let $\hat{x}=E\left(x \mid m_{1}, m_{2}\right)$ be the conditional expectation of $x$ given $m_{1}$ and $m_{2}$. The non-centrality parameter for $F$-test using $\hat{x}$ as the independent variable is

$$
\delta=n \sigma_{\hat{x}}^{2} \frac{a^{2}}{\sigma^{2}+a^{2}\left(\sigma_{x}^{2}-\sigma_{\hat{x}}^{2}\right)}
$$

where $\sigma_{x}^{2}=1 / 2$ in the F2 population and

$$
\sigma_{\hat{x}}^{2}=\frac{1}{2\left(1-r_{12}\right)}-\frac{\left(r_{1}+r_{2}\right)^{2}+4 r_{1} r_{2}\left(1-2 r_{12}-2 r_{1} r_{2}\right)}{2 r_{12}\left(1-r_{12}\right)}
$$

is the variance of $\hat{x}$ across individuals within the mapping population. The denominator of the noncentrality parameter is increased by $a^{2}\left(\sigma_{x}^{2}-\sigma_{x}^{2}\right)$, which is the inflation parameter for the residual variance due to uncertainty of the QTL genotype (Xu, 1995).

Assume that the QTL of interest sits in the middle of the interval between markers $m_{1}$ and $m_{2}$ so that $r_{1}=r_{2}=r$ and $r_{12}=2 r(1-r)$. The above variance for $\hat{x}$ is simplified to

$$
\sigma_{\hat{x}}^{2}=\frac{(1-2 r)^{2}}{2-4 r(1-r)}
$$

One can verify that when the QTL overlaps with both markers (the marker interval is infinitely small), that is, $r=0$, we get $\sigma_{x}^{2}=1 / 2$, leading to the same non-centrality parameter given in equation (3). However, if the QTL sits in the middle of an infinitely large marker interval, that is, $r=0.5$, we have $\sigma_{\hat{x}}^{2}=0$, leading to a zero non-centrality parameter and thus zero power.

Given the statistical power and the Type I error, one can find the non-centrality parameter using the following inverse function,

$$
\delta_{1-\gamma}=F_{-1}\left(\lambda_{1-\alpha} \mid 1, n, 1-\gamma\right)
$$

where $F_{-1}$ is the inverse F-distribution function with the inverse referring to the non-centrality parameter, not the quantile. We use a subscript -1 to distinguish the noncentrality inverse F-distribution function from the traditional quantile inverse F-distribution function (with a superscript -1). Once the non-centrality parameter is calculated, we can find the sample size required given the size of a marker interval or the marker interval required given a fixed sample size.

\section{Examples}

We now show a few examples for calculating the statistical power and various experimental parameters. Let us define

$$
h^{2}=\frac{\sigma_{x}^{2} a^{2}}{\sigma_{x}^{2} a^{2}+\sigma^{2}}
$$

as the proportion of the phenotypic variance explained by the QTL. The squared QTL effect can be expressed as a function of $h^{2}$, as shown below,

$$
a^{2}=\frac{h^{2}}{\sigma_{x}^{2}\left(1-h^{2}\right)} \sigma^{2}
$$

Since the squared QTL effect $a^{2}$ is only meaningful when compared with $\sigma^{2}$, we will assume $\sigma^{2}=1.0$ in all subsequent examples.

Example 1: Calculate the statistical power at a Type I error rate $\alpha=0.01$ for detecting a QTL that is located in the middle of a $20 \mathrm{cM}$ marker interval and explains $h^{2}=0.10$ of the phenotypic variance with $n=100 \quad \mathrm{~F}_{2}$ individuals.

We first need to find the recombination fraction between the QTL and the flanking markers using the Haldane (1919) mapping function,

$$
r=\frac{1}{2}[1-\exp (-2 \times 10 / 100)]=0.0906
$$

We then calculate $\sigma_{x}^{2}$ using equation (9),

$$
\sigma_{\hat{x}}^{2}=\frac{(1-2 \times 0.0906)^{2}}{2-4 \times 0.0906(1-0.0906)}=0.4013
$$

The squared QTL effect is

$$
a^{2}=\frac{0.10}{\frac{1}{2}(1-0.10)} \times 1=0.2222
$$

The non-centrality parameter is

$$
\begin{aligned}
\delta & =100 \times 0.4013 \times \frac{0.2222}{1+0.2222 \times(0.5-0.4013)} \\
& =8.7267
\end{aligned}
$$

Corresponding to the Type I error $\alpha=0.01$, the critical value for the test statistic is

$$
\lambda_{1-0.01}=F^{-1}(1-0.01 \mid 1,100,0)=6.8953
$$


The Type II error is

$$
\beta=F(6.8953 \mid 1,100,8.7267)=0.3710
$$

Therefore, the statistical power to detect such a QTL is

$$
\gamma=1-0.3710=0.6290
$$

Since the QTL is assumed in the middle of the marker interval (the worst-case scenario), this power is an underestimation of the actual power.

Example 2: Calculate the minimum marker density required to detect a QTL explaining $h^{2}=0.10$ of the phenotypic variance in an $\mathrm{F}_{2}$ mating design of $n=200$ individuals with $\gamma=0.90$ of the power under a Type I error rate $\alpha=0.01$.

The worst-case scenario is that the QTL is located in the middle of the largest marker interval. Let $D$ be the largest marker interval measured in centiMorgan. Assume that the recombination fraction between the QTL and each of the flanking markers is $r$. The recombination fraction between the two markers is $r_{12}=2 r(1-r)$. We first calculate $r$ using the method given below. We then get $r_{12}$ and convert $r_{12}$ into $D$ to obtain the largest marker interval that allows the detection of such a QTL. Given $\alpha=0.01$ and $n=200$, we found that

$$
\lambda_{1-0.01}=F^{-1}(1-0.01 \mid 1,200,0)=6.7633
$$

The non-centrality parameter is calculated using equation (10),

$$
\delta_{1-0.90}=F_{-1}(6.7633 \mid 1,200,1-0.90)=15.1299
$$

Given $\delta_{1-0.90}$, we can solve for $\sigma_{x}^{2}$ using the inverse function of equation (7),

$$
\begin{aligned}
\sigma_{\hat{x}}^{2}=\frac{\delta_{1-0.90}\left(\sigma^{2}+\sigma_{x}^{2} a^{2}\right)}{a^{2}\left(n+\delta_{1-0.90}\right)} & =\frac{15.1299 \times(1+0.5 \times 0.2222)}{0.2222 \times(200+15.1299)} \\
& =0.3516
\end{aligned}
$$

Using the reverse function of equation (9), we calculate

$$
\begin{aligned}
r & =\frac{1-\sigma_{\hat{x}}^{2}-\sqrt{\sigma_{\hat{x}}^{2}-\sigma_{\hat{x}}^{4}}}{2\left(1-\sigma_{\hat{x}}^{2}\right)} \\
& =\frac{1-0.3516-\sqrt{0.3516-0.3516^{2}}}{2 \times(1-0.3516)}=0.1318
\end{aligned}
$$

The recombination fraction between the two markers is

$$
r_{12}=2 r(1-r)=2 \times 0.1318 \times(1-0.1318)=0.2288
$$

Finally, the size of the interval measured in $\mathrm{cM}$ is

$$
\begin{aligned}
D & =-\frac{100}{2} \ln \left(1-2 r_{12}\right) \\
& =-\frac{100}{2} \ln (1-2 \times 0.2288)=30.5904
\end{aligned}
$$

Example 3: Assume that a QTL is located in the middle of a $10 \mathrm{cM}$ marker interval. Calculate the minimum sample size required to detect a QTL explaining $h^{2}=0.05$ of the phenotypic variance in an $\mathrm{F}_{2}$ mating design with power $\gamma=0.80$ under a Type I error rate $\alpha=0.01$.

Let $n$ be the sample size required to detect such a QTL. The distance between this QTL and each of the flanking markers is $5 \mathrm{cM}$, and thus $r=0.0476$ and $\sigma_{x}^{2}=0.4502$. Given $h^{2}=0.05$, we have $a^{2}=0.1053$. Therefore, the non-centrality parameter is

$$
\begin{aligned}
\delta & =n \times 0.4502 \times \frac{0.1053}{1+0.1053 \times(0.5-0.4502)} \\
& =0.0471 \times n
\end{aligned}
$$

Let us denote the threshold of the test statistic by

$$
\lambda_{1-0.01}=F^{-1}(1-0.01 \mid 1, n, 0),
$$

which is a function of $n$. We now solve the following equation with respect to $n$,

$$
0.0471 \times n=F_{-1}\left(F^{-1}(1-0.01 \mid 1, n, 0) \mid 1, n, 1-0.80\right)
$$

The solution can be found through iterations as shown below,

$$
n^{(t+1)}=\frac{1}{0.0471} F_{-1}\left(F^{-1}\left(1-0.01 \mid 1, n^{(t)}, 0\right) \mid 1, n^{(t)}, 1-0.80\right)
$$

The final solution is $n=251.7995 \approx 252$. Only seven iterations were required to converge when $n^{(0)}=100$ was used as the starting value. Therefore, we need 252 individuals for the $\mathrm{F}_{2}$ family to guarantee an $80 \%$ power to detect such a QTL.

\section{Simulation}

Although the theoretical power was derived based on solid statistical and mathematical foundation, it is always a good idea to verify the result via Monte Carlo simulation. We simulated a QTL located in the middle of a $20 \mathrm{cM}$ interval. The flanking markers have full information. Again, we set $\sigma^{2}=1$ so that $a^{2}=2 h^{2} /\left(1-h^{2}\right)$. Two levels of $h^{2}$ were used to simulate the size of the QTL, which are $h^{2}=0.05$ and $h^{2}=0.10$. The sample size varied from $n=50$ to 500 with an increment of 10 . For each simulated sample, we calculated the F-test statistic. The critical values used to declare statistical significance were chosen from the central F-distributions at $\alpha=0.05$. Under each situation, the simulation was replicated 1000 times. The empirical power under each specific situation was defined as the proportion of the replicates with the F-test statistics larger than the corresponding critical value. The empirical powers obtained from the simulations were compared with the theoretical powers, as illustrated by the plots given in Figure 1. The empirical powers fit the theoretical powers very closely, validating the theoretical derivation.

\section{Numerical evaluation}

As stated earlier, the worst situation for QTL mapping is that the QTL sits right in the middle of a marker interval. This can be verified by evaluating the power for a given QTL when the position of the QTL varies from one marker to the other marker of the interval. We assumed that the QTL explains $h^{2}=0.10$ of the phenotypic variance. The sample size was $n=100$. The marker interval was $40 \mathrm{cM}$. The position of the simulated QTL varied from $0 \mathrm{cM}$ (overlaps with the left marker) to $40 \mathrm{cM}$ (overlaps with the right marker). Figure 2 represents the power change as the position of the QTL changes within the marker interval. When the QTL overlaps with a marker, the power is about $63 \%$ (the maximum value cross the interval). The lowest power is about $43 \%$, which occurs when the QTL is located in the middle of the interval (position $20 \mathrm{cM}$ ). 


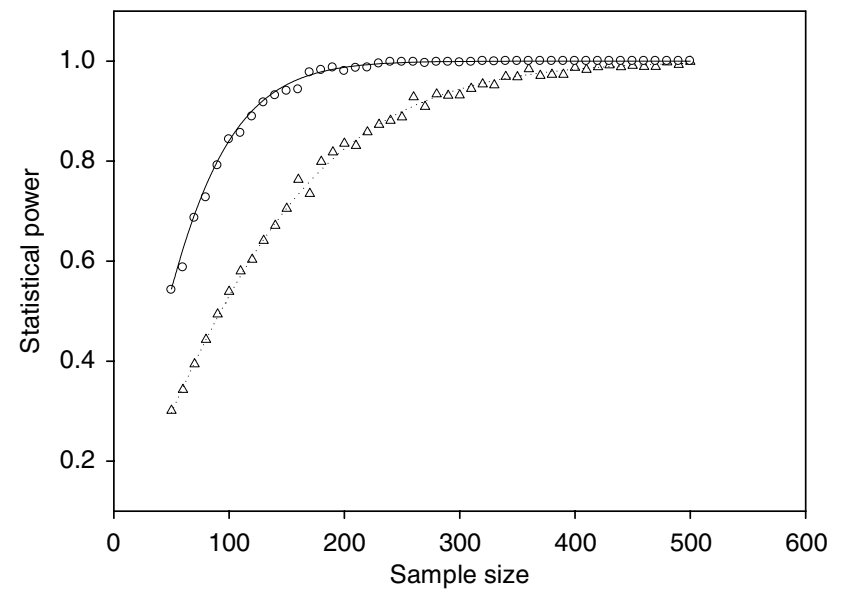

Figure 1 Comparisons of the statistical powers obtained from Monte Carlo simulations (open circles and triangles) with those predicted from the theory (dotted and solid lines). The plots with open circles and solid line (upper plots) represent the situation of $h^{2}=0.10$, while the plots with open triangles and dotted line (lower plots) represent the situation of $h^{2}=0.05$.

We now assume that the QTL of interest is located in the middle of a marker interval. We then change the size of the interval from $0 \mathrm{cM}$ (all three loci, two markers and a QTL, overlap) to $60 \mathrm{cM}$ (the QTL is located at position $30 \mathrm{cM}$ ). We also allow the sample size to vary from $n=50$ to $n=1000$. We evaluated the power under two levels of the QTL size, $h^{2}=0.05$ and $h^{2}=0.10$. The power surfaces are plotted in the 3D graph shown in Figure 3. The following three conclusions were obtained from the result. (1) Increase of the sample size can significantly improve the statistical power. Although this conclusion is trivial, it serves as a partial validation for the formula to calculate the statistical power. When the QTL overlaps with the markers and the population is 200 or more, we will have high confidence $(90 \%)$ to detect a small QTL that only contributes $5 \%$ of the phenotypic variance. (2) Change of interval size also affects the statistical power. When the interval size is increased from 0 to $60 \mathrm{cM}$, the power for detection of the same QTL is decreased from 100 to $60 \%$. The effect of marker interval size on the power is certainly less than the effect of the sample size on the power. (3) Neither a large sample size nor a high marker density is absolutely necessary for detecting a large QTL, for example, when the QTL contributes $10 \%$ or more of the phenotype variance, the theoretical power is as high as 60\% even if the QTL is in the middle of a large interval $(60 \mathrm{cM})$ and only 100 individuals are used in the experiment.

\section{Discussion}

Simple methods for calculating the statistical power apply to other mating designs of line crossing experiments. As long as the genotype indicator variables are defined in the same scale for all experiments, the powers can be compared. Here, we extend the power calculation to the following mating designs: $\mathrm{BC}$ (backcross), $\mathrm{DH}$ (double haploid) and RIL (recombinant inbred line). The additive genetic effect (a) in all cases is defined as the difference between two genotypes that differ by only one allele. If two genotypes differ by two alleles, the

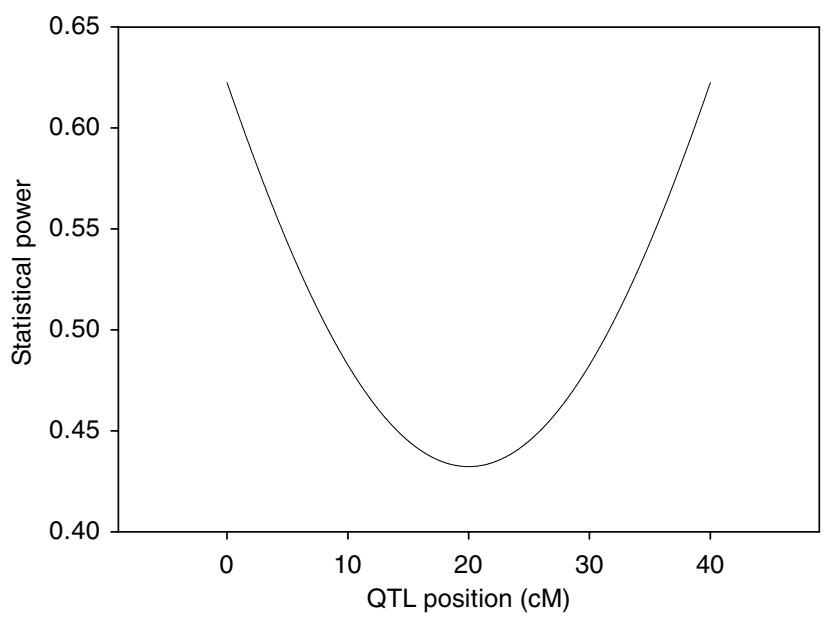

Figure 2 Changes of statistical power as QTL position changes from one end to the other end of a marker interval of $40 \mathrm{cM}$ in length. The sample size is $n=100$ and the size of the QTL is $h^{2}=0.05$. The lowest power occurs when the QTL is in the middle of the marker interval.

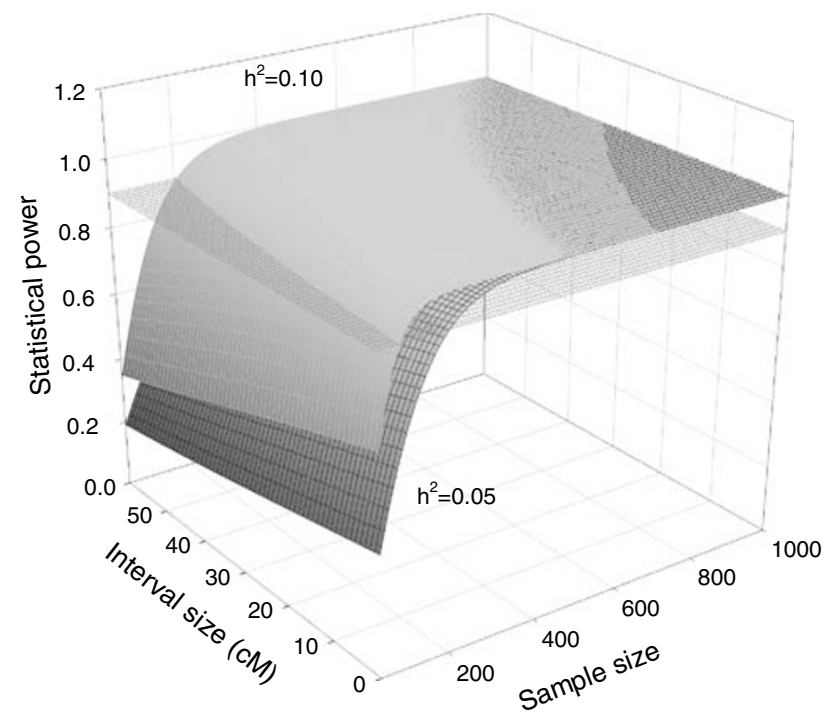

Figure 3 Change of statistical power as the sample size and marker interval change. The gray surface (upper) represents the statistical power under $h^{2}=0.10$ while the surface with dark grids (lower) represents the statistical power under $h^{2}=0.05$. The horizontal plane represents the power of $\gamma=0.90$.

difference is expressed by $2 a$. Different mating designs have different powers because they have different noncentrality parameters.

\section{$\mathrm{BC}$ design}

There are only two genotypes, $A_{1} A_{1}$ and $A_{1} A_{2}$. Therefore, the $x$ variable is defined as $x=1 / 2$ and $x=-1 / 2$, respectively, for the two genotypes. The true variance of $x$ is $\sigma_{x}^{2}=1 / 2$. This leads to the following variance of $\hat{x}$,

$$
\sigma_{\hat{x}}^{2}=\frac{(1-2 r)^{2}}{4-8(1-r) r}
$$


where $r$ is the recombination fraction between the QTL and a flanking marker (the QTL is in the middle of the two flanking markers).

\section{$\mathrm{DH}$ design}

The two genotypes in a DH family are $A_{1} A_{1}$ and $A_{2} A_{2}$. Therefore, the $x$ variable is defined as $x=1$ and $x=-1$, respectively, for the two genotypes. The variance of $x$ is $\sigma_{x}^{2}=1$. The variance of $\hat{x}$ is

$$
\sigma_{\hat{x}}^{2}=2-\frac{1}{1-2(1-r) r}
$$

\section{RIL design}

The two genotypes for an RIL family are $A_{1} A_{1}$ and $A_{2} A_{2}$. Therefore, the $x$ variable is defined as $x=1$ and $x=-1$, respectively, for the two genotypes. The variance of $x$ is $\sigma_{x}^{2}=1$. The variance of $\hat{x}$ is

$$
\sigma_{\hat{x}}^{2}=1-\frac{4 r}{1+4 r^{2}}
$$

Current methods for power calculation are primarily based on the assumption that the QTL overlaps with a fully informative marker (Soller et al., 1976; Muranty, 1996; Lynch and Walsh, 1998). When the QTL does not overlap a marker, that is, it is away from a marker. The power can still be calculated using the nearest marker (Soller and Genizi, 1978; Lynch and Walsh, 1998). This is a single marker analysis. However, interval mapping that uses flanking marker is more efficient than the single marker analysis, and power calculation using flanking markers has not been developed. This study explores the possibility of using a simple method to calculate the statistical power of QTL detection using flanking markers. The method has been verified using the simulated data.

The two flanking markers are assumed to be fully informative. In situations where they are not fully informative, we the estimated QTL genotype $\sigma_{x}^{2}$ will be further reduced. A reduction of $\sigma_{x}^{2}$ will lead to decreased power. For example, if $5 \%$ of the individuals have missing marker genotypes, the reduced variance of $\hat{x}$ will be $0.05 \times 0+0.95 \times \sigma_{\hat{x}}^{2}=0.95 \sigma_{\hat{x}}^{2}$, where $\sigma_{\hat{x}}^{2}$ is the variance of $\hat{x}$ when no missing marker genotype is assumed. The reason for this is that when an individual has missing genotypes, the variance of $\hat{x}$ is zero, that is, $\sigma_{\hat{x}}^{2}=0$.
When genome scanning is performed, multiple tests are involved. A reasonable alpha-value for a genomewide QTL analysis was suggested by Muranty, 1996, which is $0.001 \leqslant \alpha \leqslant 0.01$.

We have developed a SAS program to calculate the statistical power using the simple method. The following parameters are considered in the power calculation: the power $(\gamma)$, the Type I error $(\alpha)$, the size of the QTL $\left(h^{2}\right)$, the sample size $(n)$, the size of the marker interval $(D)$ denoted as distance between a marker measured in $\mathrm{cM}$ and the type of line cross (F2, BC, DH and RIL). For a given type of line cross, the program can compute one parameter given the remaining four parameters. The program is available on request from the authors or downloadable from our website www.statgen.ucr.edu.

\section{Acknowledgements}

This project is supported by the National Research Initiative Plant Genome Program of the USDA Cooperative State Research, Education and Extension Service 2007-02784 to SX.

\section{References}

Dupuis J, Siegmund D (1999). Statistical methods for mapping quantitative trait loci from a dense set of markers. Genetics 151: 373-386.

Haldane JBS (1919). The combination of linkage values and the calculation of distances between the loci of linked factors. J Genet 8: 299-309.

Haley CS, Knott SA (1992). A simple regression method for mapping quantitative trait loci in line crosses using flanking markers. Heredity 69: 315-324.

Lynch M, Walsh B (1998). Genetics and Analysis of Quantitative Traits. Sinaure Associates Inc.: Sunderland, Massachusetts.

Muranty H (1996). Power of tests for quantitative trait loci detection using full-sib families in different schemes. Heredity 76: 156-165.

Soller M, Brody T, Genizi A (1976). On the power of experimental designs for the detection of linkage between marker loci and quantitative loci in crosses between inbred lines. Theor Appl Genet 47: 35-39.

Soller M, Genizi A (1978). The efficiency of experimental designs for the detection of linkage between a marker locus and a locus affecting a quantitative trait in segregating populations. Biometrics 34: 47-55.

Xu S (1995). A comment on the simple regression method for interval mapping. Genetics 141: 1657-1659. 